\title{
The Maximal Oxygen Uptake Verification Phase: a Light at the End of the Tunnel?
}

\author{
Gustavo Z. Schaun
}

\begin{abstract}
Commonly performed during an incremental test to exhaustion, maximal oxygen uptake $\left(\mathrm{V}_{2 \text { max }}\right)$ assessment has become a recurring practice in clinical and experimental settings. To validate the test, several criteria were proposed. In this context, the plateau in oxygen uptake $\left(\dot{\mathrm{V}}_{2}\right)$ is inconsistent in its frequency, reducing its usefulness as a robust method to determine "true" $\dot{\mathrm{V}}_{2 \max }$. Moreover, secondary criteria previously suggested, such as expiratory exchange ratios or percentages of maximal heart rate, are highly dependent on protocol design and often are achieved at $\dot{\mathrm{V}}_{2}$ percentages well below $\dot{\mathrm{V}}_{2 \max }$. Thus, an alternative method termed verification phase was proposed. Currently, it is clear that the verification phase can be a practical and sensitive method to confirm $\dot{\mathrm{V}} \mathrm{O}_{2 \text { maxi }}$ however, procedures to conduct it are not standardized across the literature and no previous research tried to summarize how it has been employed. Therefore, in this review the knowledge on the verification phase was updated, while suggestions on how it can be performed (e.g. intensity, duration, recovery) were provided according to population and protocol design. Future studies should focus to identify a verification protocol feasible for different populations and to compare square-wave and multistage verification phases. Additionally, studies assessing verification phases in different patient populations are still warranted.
\end{abstract}

Keywords: Exercise, Oxygen consumption, Incremental exercise, Graded exercise test, Cardiopulmonary exercise testing, Plateau, Criteria

\section{Key Points}

$>$ Both supramaximal and submaximal protocols appear to be suitable for measuring maximal oxygen consumption $\left(\dot{\mathrm{V}} \mathrm{O}_{2 \max }\right)$, while verification phase durations between 2 and $3 \mathrm{~min}$ should already be adequate to confirm $\dot{\mathrm{VO}}_{2 \max }$ for most, but not all, subjects.

$>$ Based on available data, recovery time between phases does not appear to be critical, although more research is warranted, and processing data with 15- to 30-s time averages seems reasonable.

$>$ When all safety measures are taken, the verification phase seems to be well tolerated in both healthy and patient populations in general. Nevertheless, more research is still necessary to further confirm verification phase applicability in older adults and clinical populations, as well as in longitudinal studies.

Correspondence: gustavoschaun@hotmail.com

Neuromuscular Assessment Laboratory, Physical Education School, Federal University of Pelotas, Rua Luís de Camões, 625, Tablada, Pelotas, RS 96055-630, Brazil

\section{Review}

Background

Since its identification in the 1920s, the maximal oxygen uptake $\left(\dot{\mathrm{V}}_{2 \max }\right)$ has gained substantial clinic and scienrelevance. Initially proposed by Hill and Lupton [1] $\mathrm{O}_{2 \max }$ represents a physiological ceiling on the capacity to increase alveolar $\mathrm{O}_{2}$ uptake, $\mathrm{O}_{2}$ transport and/or conintion at tissue level in response to an increase in toad and metabolic demand. Currently, it is accepted as the gold standard to assess cardiorespiratory fitness [2] and is also one of the best individual predictors of all-cause mortality and cardiovascular disease risk [3-5]. Consequently, a sufficiently high $\mathrm{VO}_{2 \max }$ is imquality of life in the general population.

$\dot{\mathrm{V}} \mathrm{O}_{2 \max }$ has also become relevant within the field of exercise performance. In endurance sports such as running, cycling and cross-country skiing, for example, a high $\dot{\mathrm{V}}_{2 \max }$ is a conditioning variable for sporting success [6]. In other sports, like football and rugby, high cardiorespiratory fitness may assist recovery 
between the brief high-intensity efforts, characteristic of these modalities [7, 8]. Moreover, several physiologists and conditioning coaches use variables associated with $\dot{\mathrm{V}}_{2 \max }$ (e.g. velocity, power, heart rate) to design and prescribe training programs. The efficiency of these programs to enhance cardiorespiratory fitness can also be determined by $\dot{\mathrm{V}} \mathrm{O}_{2 \max }$ reassessment.

Accordingly, valid and reliable tests (i.e. capable of being replicated) are necessary to proper evaluate $\dot{\mathrm{V}}_{2 \max }$. Over the years, the most commonly used protocol is the incremental load test performed as a progressive ramp or from step increments until exhaustion [9]. Protocol characteristics, such as the initial velocity/power and incline, workload increments, stage duration and ergometer, are designed according to the population being assessed to allow $\dot{\mathrm{VO}}_{2 \max }$ to be properly achieved. However, a question that continues to intrigue researchers and scientists for decades is whether, in fact, it is possible to attest that the $\dot{\mathrm{V}} \mathrm{O}_{2 \max }$ measured is a real maximal value. For this purpose, the plateau in $\dot{\mathrm{VO}}_{2}$ stands out as the main criterion employed [10-12]. As will be discussed in an appropriate session, a plateau is not always identified in all tested individuals. Hence, several secondary criteria were proposed [13], which have been extensively criticized in recent years for their lack of validity and sensitivity [2, 10-12, 14-17].

Thereafter, an alternative solution termed "verification phase" was proposed [18]. In summary, after the incremental test, a new effort is performed and $\dot{\mathrm{V}}_{2 \text { max }}$ results are compared between phases. The validity of this procedure has already been confirmed in eutrophic and obese adults, patient with clinical conditions and children [12]. Midgley and colleagues, in 2007, published an article in Sports Medicine suggesting that future studies should focus on investigating the verification phase protocols and how to improve their utility and validity [14]. Approximately 10 years later, it is important to assess scientific findings that have followed regarding the verification phase and whether or not there are issues that still require further attention. Thus, the aim of the present review was to provide an update on the knowledge produced on the $\dot{V} \mathrm{O}_{2 \max }$ verification phase, highlighting the advances in methodology and suggesting future directions for research. Furthermore, although previous research has confirmed the usefulness of this phase and has clarified the rationale for its incorporation [12], no investigation tried to summarize how it has been done while also providing clear recommendations on how the verification phase should be performed as a whole (e.g. intensity, duration, protocol design, test validation). Hence, the present paper should assist researchers, as well as professionals, to employ the verification phase more adequately. Due to the great heterogeneity between protocols and among studied populations, the author considered it inappropriate to perform a systematic review and meta-analysis.

\section{$\dot{\mathrm{VO}}_{2}$ Plateau-Fool's Gold?}

The notion of a reduction in the $\mathrm{O}_{2}$ uptake slope near the end of the incremental test, despite a progressive increase in intensity, also seems to have been introduced by Hill and his colleagues [1, 19], although this is not a consensus view [20]. Regardless, this plateau in oxygen uptake $\left(\dot{\mathrm{VO}}_{2}\right)$ is understood as the best evidence that a "true" $\dot{\mathrm{V}} \mathrm{O}_{2 \max }$ was reached during the incremental test [9]. However, more than 80 years have passed since Hill's pioneering studies and the answer to which would be the definitive criterion to confirm the occurrence of a plateau has not yet been given. Taylor et al. have possibly suggested what came to be the most popular criterion in the recent decades for this purpose [21]. These authors concluded that changes $\leq 150 \mathrm{ml} \mathrm{min}{ }^{-1}$ between two consecutive stages meant that it could "[...] safely be assumed that a maximal oxygen uptake [...]" had "[...] been attained", that is, values $\leq 50 \%$ of the expected increase. On this regard, Taylor and colleagues were able to demonstrate a plateau in 108 out of 115 tests (i.e.

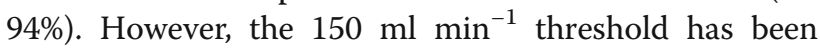
criticized because of its lack of theoretical and statistical basis, as well as its lack of specificity in relation to the protocols currently used [22]. Investigations that attempted to replicate Taylor's findings found a great variability in the percentage of subjects who presented a plateau in $\dot{\mathrm{V}}_{2}[23,24]$.

Moreover, other threshold values have also been adopted, such as $\leq 100 \mathrm{ml} \mathrm{kg}$ or $\leq 50 \mathrm{ml} \mathrm{kg}^{-1}$ of $\mathrm{O}_{2}$ $[22,25]$ making it even more difficult to compare studies. Regardless of the threshold adopted, a strong inconsistency as to the number or percentage of plateaus observed is found in the literature [20, 26]. Previous studies have identified 100\% [22] and 94\% [21] plateau incidence, while others reported values as low as $47 \%$, 24\% [27], 17\% [28] and even 0\% [29]. Beltz et al. [2] emphasize that age, modality tested and how data are processed are among the main factors that can influence the incidence of plateaus (for a detailed review on data processing, see Robergs et al. [30]). Physical fitness and incremental test protocol are factors that can also influence the incidence of plateaus in $\mathrm{VO}_{2}[11,31]$. As stated by Midgley and Carroll [11], together, these considerations reduce the usefulness of the plateau criterion as a robust method for determining a "true" $\mathrm{V}_{2 \max }$, not because of the plateau itself, but by the methodology used in its identification.

At present, there seems to be a consensus in the literature regarding the existence of the plateau phenomenon in $\dot{\mathrm{VO}}_{2}[20,22,26]$. Nevertheless, some recent studies have questioned the necessity of this phenomenon to 
characterize that a $\dot{\mathrm{V}}_{2 \max }$ has been reached $[15,26,28]$. After submitting 52 well-trained distance runners to an incremental treadmill test and a second bout to exhaustion at an intensity 30\% higher than the incremental phase, Hawkins and colleagues [32] found no difference between $\dot{\mathrm{VO}_{2 \max }}$ in both tests. Similarly, eight welltrained men underwent two maximal and one supramaximal test on a combined arm and leg exercise [33]. No differences were observed in $\dot{\mathrm{V}}_{2 \max }$, contributing to the notion that actually there is a limit on $\dot{\mathrm{VO}}_{2}$ (see also Day et al. [28]). Furthermore, it should be noted that the same subject who performs an incremental test twice can show a plateau in only one of them while presenting similar $\dot{\mathrm{V}} \mathrm{O}_{2 \max }$ values between the tests $[15,34,35]$. Therefore, although the plateau is still considered the best evidence to confirm that a $\dot{\mathrm{V}} \mathrm{O}_{2 \max }$ has been reached, is the effort employed to identify this phenomenon worth it considering its inconsistency?

\section{Secondary Criteria}

Based on these assumptions, researchers deemed necessary to define secondary criteria for when a $\mathrm{VO}_{2}$ plateau was not evident [36]. The most common ones are thresholds in the respiratory exchange ratio (RER); agepredicted maximal heart rate $\left(\mathrm{HR}_{\max }\right)$; blood lactate concentration; and on Borg's rating of perceived effort scale (RPE) $[13,17,24,36]$. The rationale is that in the absence of an evident plateau, researchers could attest that a maximal effort was given [36, 37]. However, some subjects do not reach the criteria even when a maximum effort was given. On the other hand, these criteria also often end up including subjects who did not perform maximally and, consequently, underestimates $\dot{\mathrm{V}}_{2 \max }$. Poole et al. [17] showed in eight healthy men that more liberal thresholds, such as $\pm 10 \mathrm{bpm}$ of the age-predicted $\mathrm{HR}_{\max }$ and $\mathrm{RER} \geq 1.10$, were already reached at 75 to $80 \%$ of the $\dot{\mathrm{V}}_{2 \max }$. Experimental studies employing these criteria may underestimate $\dot{\mathrm{VO}}_{2 \text { max }}$ during baseline testing and, in turn, overestimate $\dot{\mathrm{V}}_{2 \max }$ increase after the intervention, for example.

Finally, and perhaps more importantly, contrary to what would be expected, these criteria are also not able to differentiate those who demonstrate a plateau in $\dot{\mathrm{VO}}_{2}$ from those who do not [17,31]. After an incremental test on a cycle ergometer, only two out of 99 adults presented a plateau in $\dot{V}_{2}, R E R \geq 1.15$ and reached \pm 10 bpm of the age-predicted $\mathrm{HR}_{\max }$ [16]. In addition, these cutoff points can be decided a posteriori, that is, researchers can choose the most convenient value based on the results obtained. These considerations led previous authors to suggest the complete rejection of secondary criteria to validate maximal tests [11, 17]. Yet, in 2010, a survey encompassing approximately 75 subjects trained in the field of exercise physiology showed that $52 \%$ performed their $\dot{\mathrm{V}}_{2 \max }$ assessments and data processing based on subjective concepts such as "beliefs and traditions" [30]. Therefore, greater efforts are needed to identify alternative methods and to popularize these alternatives in the clinical and scientific community. Only then will inadequate criteria cease to be employed.

\section{Verification Phase: a Light at the End of the Tunnel?}

So far, we have seen that the incidence of a plateau in $\dot{\mathrm{V}} \mathrm{O}_{2}$ during incremental tests is or may be low. Additionally, the secondary criteria used, at least those mentioned above, present great inter-subject variability compromising the sensitivity and reliability of these criteria (see the "Secondary Criteria" section). As a consequence, a viable alternative to confirm true $\dot{\mathrm{VO}}_{2 \max }$ still need to be identified. Dating from at least 1982, based on a book chapter written by Thoden and colleagues, the "exhaustive phase", which would later be renamed to "verification phase" [18], proposed a second effort at an intensity higher than the incremental test to be performed $[2,11]$. The first scientific report on the use of verification phases seems to be that of Niemelä et al. [16]. The authors assessed 16 healthy men who performed an incremental ramp test on a cycle ergometer and 1 week later underwent a warm-up comprising one or two submaximal workloads followed by supramaximal exertion. Additionally, Morgan et al.'s [38] study was suggested as the primary study to incorporate the verification phase as part of its methods [11]. Highly trained runners completed a maximal treadmill test and those who did not show a plateau in $\dot{\mathrm{VO}}_{2}$ during the test performed a four-minute supramaximal effort, $10 \mathrm{~min}$ after the incremental test. Both studies found no differences between the means in $\dot{\mathrm{V}}{ }_{2 \max }$ from the incremental test and the verification phase. Although there are some caveats regarding the methodology and data reporting of these studies, it is undeniable that both provided promising results and demonstrated that the use of the verification phase could be a viable method to confirm $\dot{\mathrm{V}} \mathrm{O}_{2 \max }$ (for a historical perspective on the verification phase, readers are referred to Beltz et al. [2] and Midgley and Carroll [11]).

Therefore, it is important to define what exactly is configured as a verification phase. According to Pettit et al. [39], it is an exhaustive square-wave effort used to corroborate $\dot{\mathrm{V}}_{2 \max }$ measured during incremental testing. For Midgley et al. [35], the verification phase is characterized as a recovery of 5-15 min after the completion of the progressive test followed by an effort to exhaustion one stage higher than the progressive test. However, there are studies that perform the verification phase on a separate day [40] and at intensities lower than maximal [29, 41, 42]. Moreover, some use a square-wave design while others use a multistage approach (Fig. 1). Thus, a more 


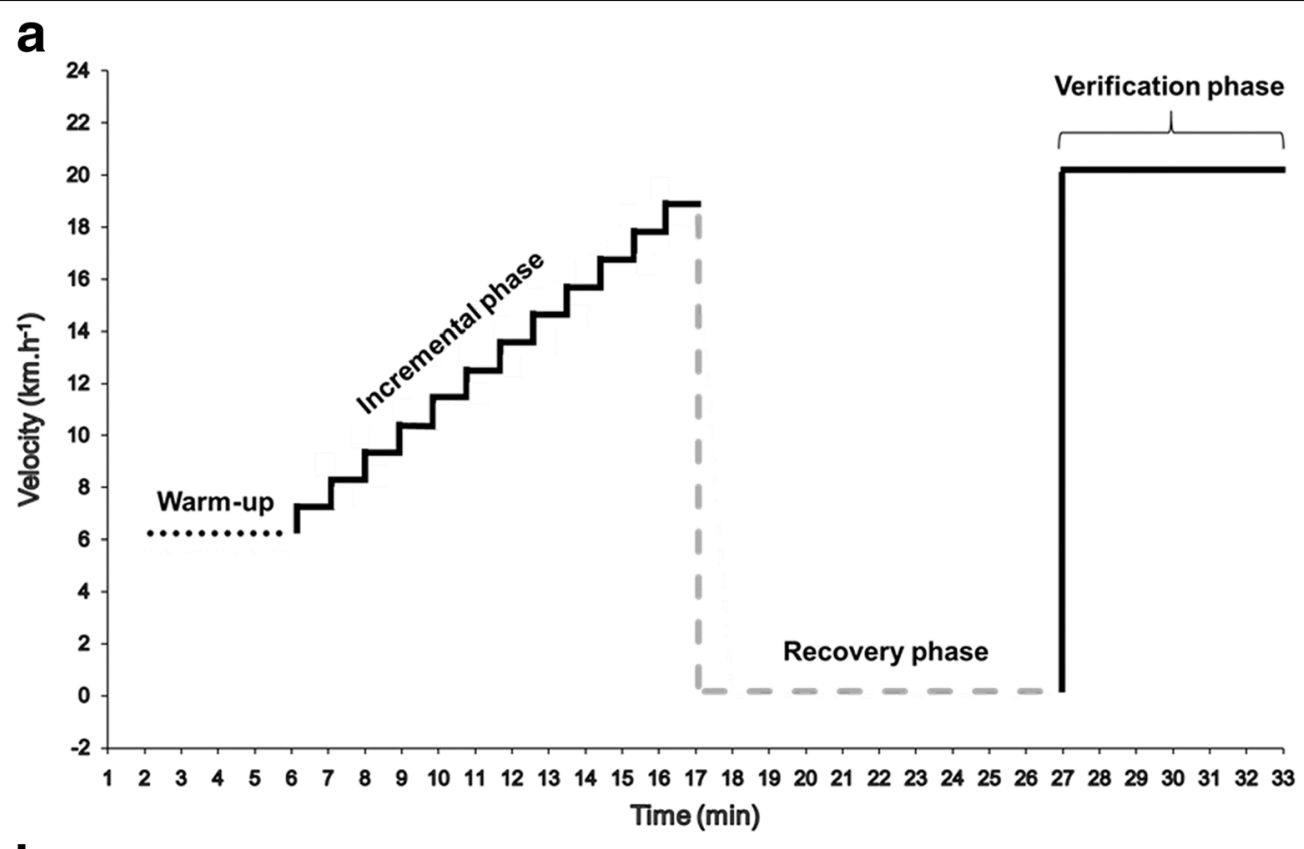

b

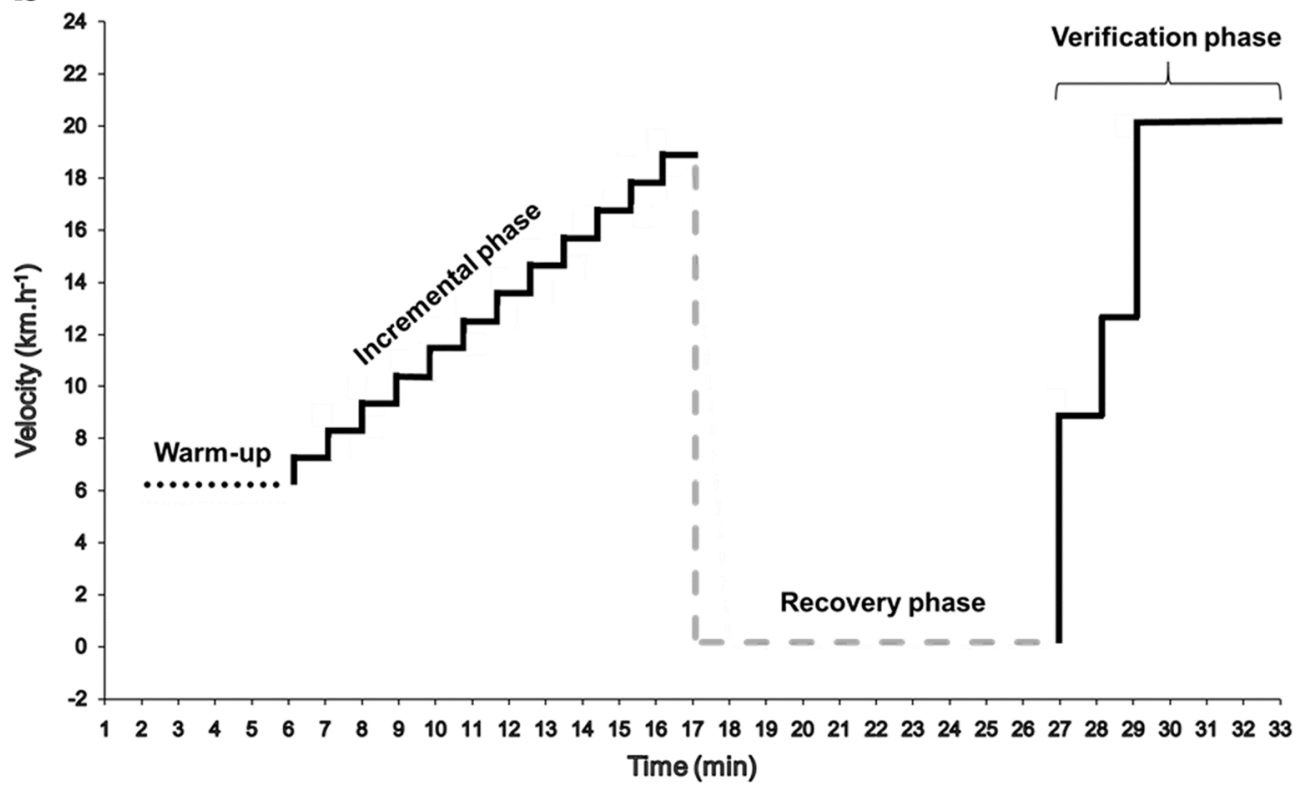

Fig. 1 Schematic illustration of an incremental test followed by either a square-wave (a) or a multistage (b) verification phase. Incremental protocol consists of a 5-min warm-up followed by $1 \mathrm{~km} \mathrm{~h}^{-1}$ increments every minute until exhaustion (i.e. $19 \mathrm{~km} \mathrm{~h}^{-1}$ ). After 10 min of passive recovery, an effort at one stage higher $\left(20 \mathrm{~km} \mathrm{~h}^{-1}\right)$ than the last stage completed during the incremental phase is performed directly (a) or preceded by a "warm-up" corresponding to 2 min at 50\% and $1 \mathrm{~min}$ at 70\% of the maximal velocity reached during the incremental phase (b)

comprehensive definition encompassing these characteristics becomes necessary. In a broad sense, the verification phase is an effort performed after an incremental test to exhaustion, in the same session or not, with intensities ranging from submaximal (above critical power) to supramaximal that allow sufficient time for $\dot{\mathrm{VO}}_{2 \max }$ to be reached. The $\dot{\mathrm{V}}_{2 \max }$ measured during the verification phase is then compared to that of the incremental phase and if both do not differ, based on a given criterion, the test is considered valid and $\dot{\mathrm{VO}}_{2 \max }$ as true.

Although the verification phase has been suggested and investigated for almost 30 years, not many studies applied this method in their investigation [37]. It was suggested that the reason for this would be the lack of studies supporting its validity [11]. However, in recent years several experiments have been carried out showing 
that the verification phase can confirm true $\dot{\mathrm{V}}_{2 \max }$ in different populations ranging from highly trained athletes [32, 43], recreationally trained subjects [41, 42], physically active [44], sedentary [31] and clinical populations $[45,46]$. These studies along with their protocols are presented in Table 1. Accordingly, the next sections will seek to summarize how the verification phase has been performed and provide suggestions for its implementation whenever possible.

\section{How It Should Be Done-Is There Consensus? Intensity}

As stated in the previous section, intensities employed during the verification phase ranged from submaximal to supramaximal efforts. Specifically, intensities between 90 and $130 \%$ of those associated with $\dot{\mathrm{VO}}_{2 \max }\left(\mathrm{i} \dot{V} \mathrm{O}_{2 \text { max }}\right)$ have already been used (Table 1), and there is still no consensus as to the correct option, if there is one. According to some authors, maximal and submaximal intensities would not incorporate the original plateau concept, indicated by the absence of increment in $\dot{\mathrm{VO}}_{2}$ versus an increase in exercise intensity [14]. On the other hand, it seems that exercise intensities above the critical power would already be enough to evoke $\dot{\mathrm{VO}}_{2 \max }$ [39] provided that exercise duration is sufficient. Day et al. [28] submitted 71 healthy men to a verification phase at $90 \% \mathrm{iVO}_{2 \max }$ on a cycle ergometer and found no difference in the mean $\dot{\mathrm{V}} \mathrm{O}_{2 \max }$ between incremental and verification phases. Similarly, Kirkeberg et al. [41] assessed 12 recreationally trained men at two stages previous to that reached at the end of the incremental test, and no difference in $\dot{\mathrm{VO}}_{2 \max }$ was found as well. More recently, one study attempted to compare distinct verification phases, one at $105 \%$ of the peak work rate and another at two stages prior to the end of the incremental test [42]. The authors concluded that both verification phases appeared to be valid and no differences were found between them. Comparable results were also reported by Rossiter et al. [29] between verification phases at 105 and $95 \%$ of the peak work rate.

Nonetheless, some aspects can be observed in order to select an appropriate intensity. Steeper incremental protocols and short duration stages (e.g. $1 \mathrm{~min}$ ) tend to result in higher peak workloads at the end of the test, while less steep protocols or longer stages (e.g. $3 \mathrm{~min}$ ) terminate at workloads closer to critical power $[39,47]$. Considering that the verification phase is usually performed based on the peak velocity or power attained at the incremental test, protocols with longer or less steep stages followed by submaximal verification phases could end up using intensities below the critical power for some subjects. Conversely, shorter or steeper stages could result in very high intensities and very short verification phases, making it impossible for certain subjects to reach $\dot{\mathrm{V}} \mathrm{O}_{2 \max }$ before exhaustion $[2,11]$. This led some authors to suggest that incremental tests encompassing shorter stages and longer stages were confirmed by submaximal and supramaximal intensities, respectively [39]. However, in general both supramaximal and submaximal protocols appear to be able to confirm $\dot{\mathrm{V}}{ }_{2 \max }$ on most occasions to a greater degree than other criteria $[17,28,29,40,41,48]$ (readers are also referred to Additional file 1: Table S1).

Finally, researchers, physiologists and clinicians should keep in mind that the primary goal of the phase is not simply to achieve a $\dot{\mathrm{VO}}_{2}$ similar to the incremental phase. Rather, the goal is to create a platform that enables a higher $\mathrm{VO}_{2}$ to be reached if it has not been reached previously (Fig. 2). Thus, the intensity selected should be sufficient to generate increments (or differences) in $\dot{\mathrm{VO}}_{2}$ greater than the total measurement error (this topic will be addressed in the "Data Processing" section).

\section{Duration}

Another important aspect that can interfere with the result of the verification phase is its duration or, more precisely, the time to exhaustion during the phase. This means that the duration of the verification phase should be sufficient to allow $\dot{\mathrm{V}}_{2 \max }$ to be reached or evoked. In addition, it means that the duration of the phase is closely related to the intensity employed. Recently, it has been suggested that the time to exhaustion in the intensities mentioned in the previous section is between 3 and 6 min [12]. Still, several studies reported shorter times to exhaustion during their verification phases [29, 35, 40, 42, 43, 45, 49-53]. Rossiter et al. [29] reported time to exhaustion of only 1.47 min with a mean difference between incremental and verification phase $\dot{\mathrm{V}} \mathrm{O}_{2 \max }$ of only $31 \mathrm{ml} \mathrm{min}^{-1}$ (i.e. less than $1 \%$ ). Sawyer et al. [53] reported $1.91 \mathrm{~min}$ in sedentary obese and found no relationship between the duration of the verification phase and the difference between incremental test and verification phase $\dot{\mathrm{V}}_{2 \max }$. Furthermore, another previous study demonstrated that shorter times to exhaustion did not systematically influence the ratio between verification phase and incremental test $\dot{\mathrm{V}} \mathrm{O}_{2 \text { peak }}$ in para-athletes who had been able to exercise for more than $100 \mathrm{~s}$ [52]. However, for those who exercised for $90 \mathrm{~s}$ or less, duration seemed to exert influence. Therefore, the $\dot{\mathrm{VO}}_{2}$ kinetics of the population being assessed should be taken into account when planning or analysing the verification phase duration [11, 54]. That is, untrained subjects, older individuals, or patients with chronic heart failure, for example, may require a relatively greater time to achieve $\dot{\mathrm{V}}_{2 \max }$ compared to healthy, active, or trained subjects. Notwithstanding, considering the intensities commonly applied, this 


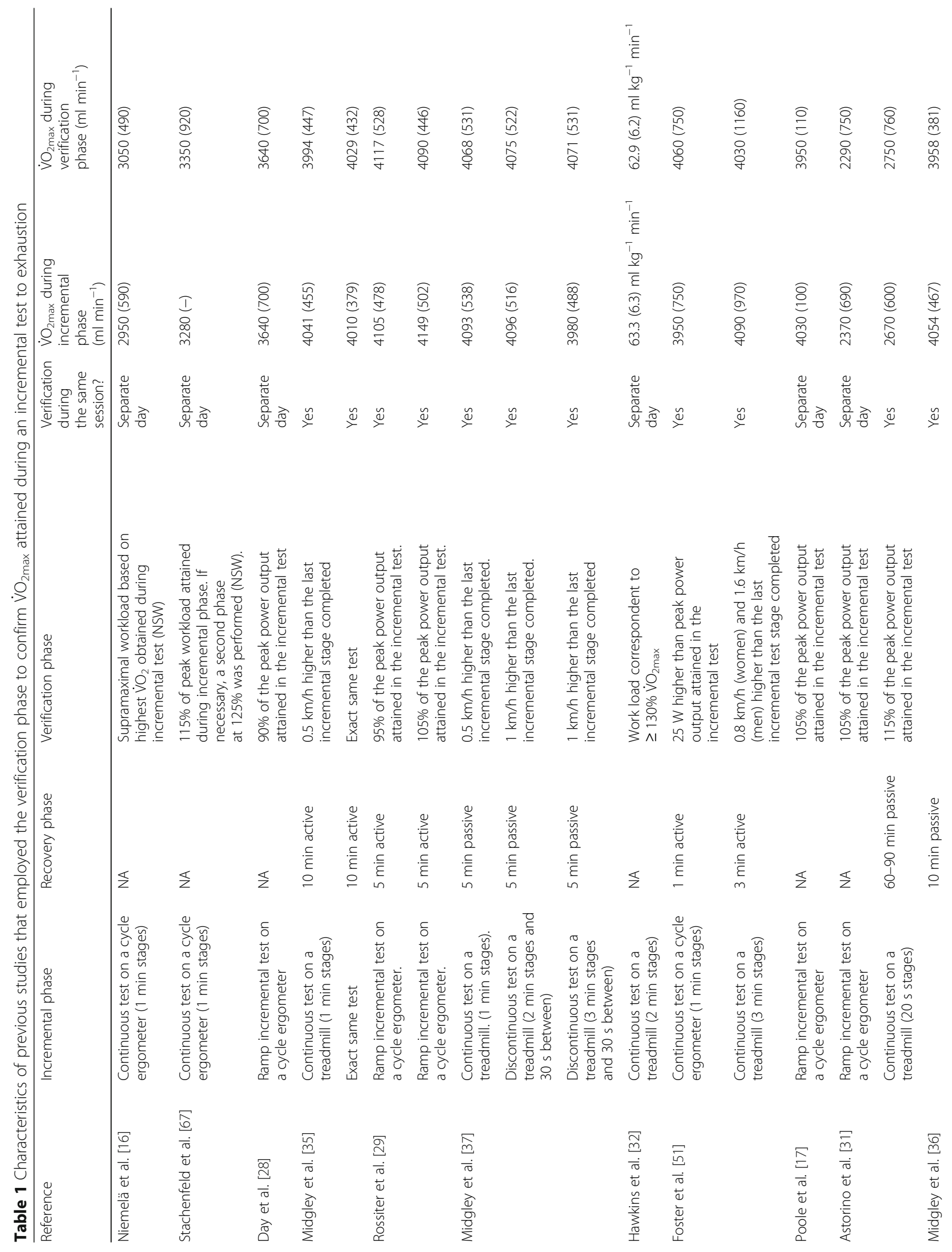




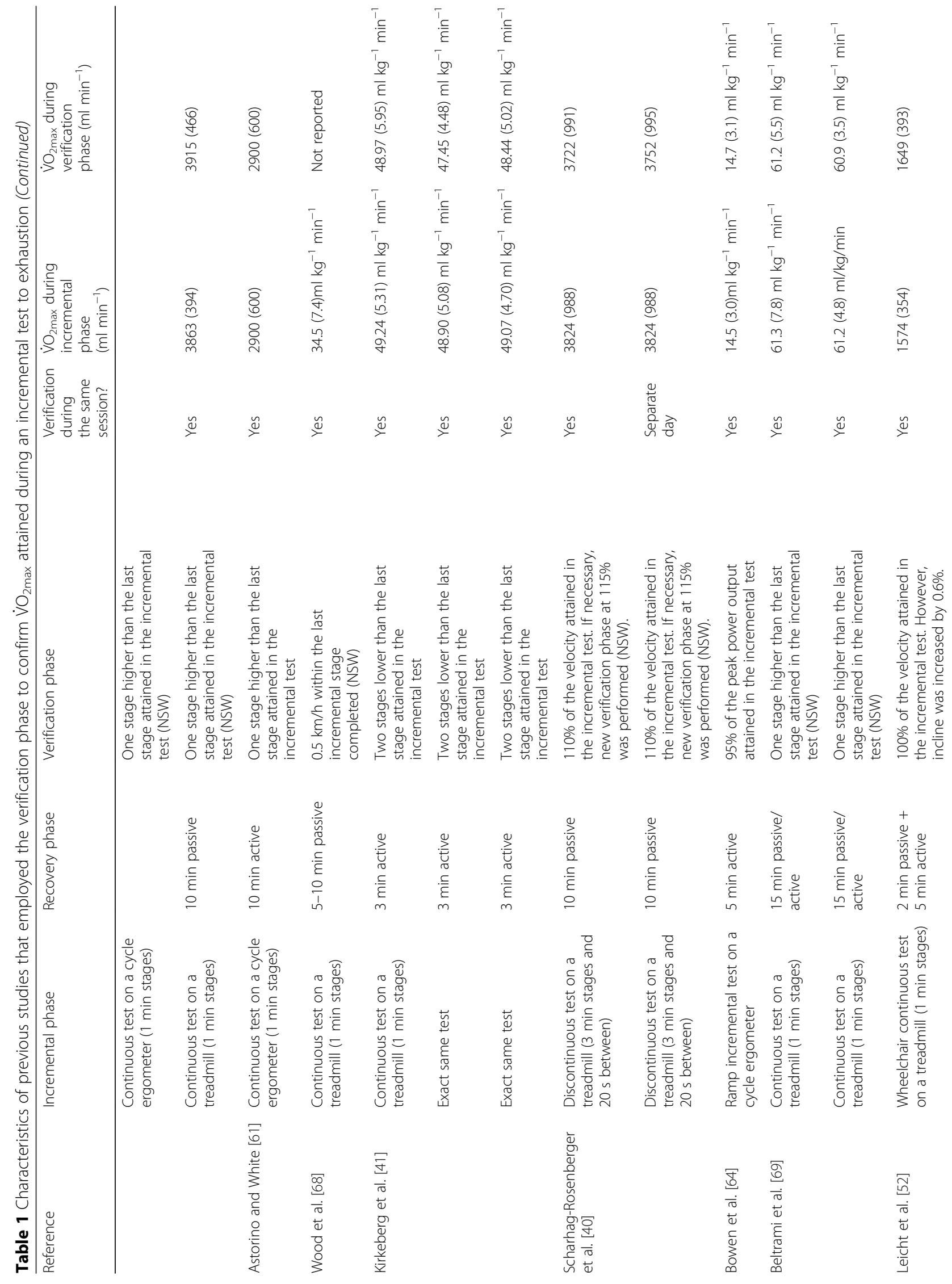




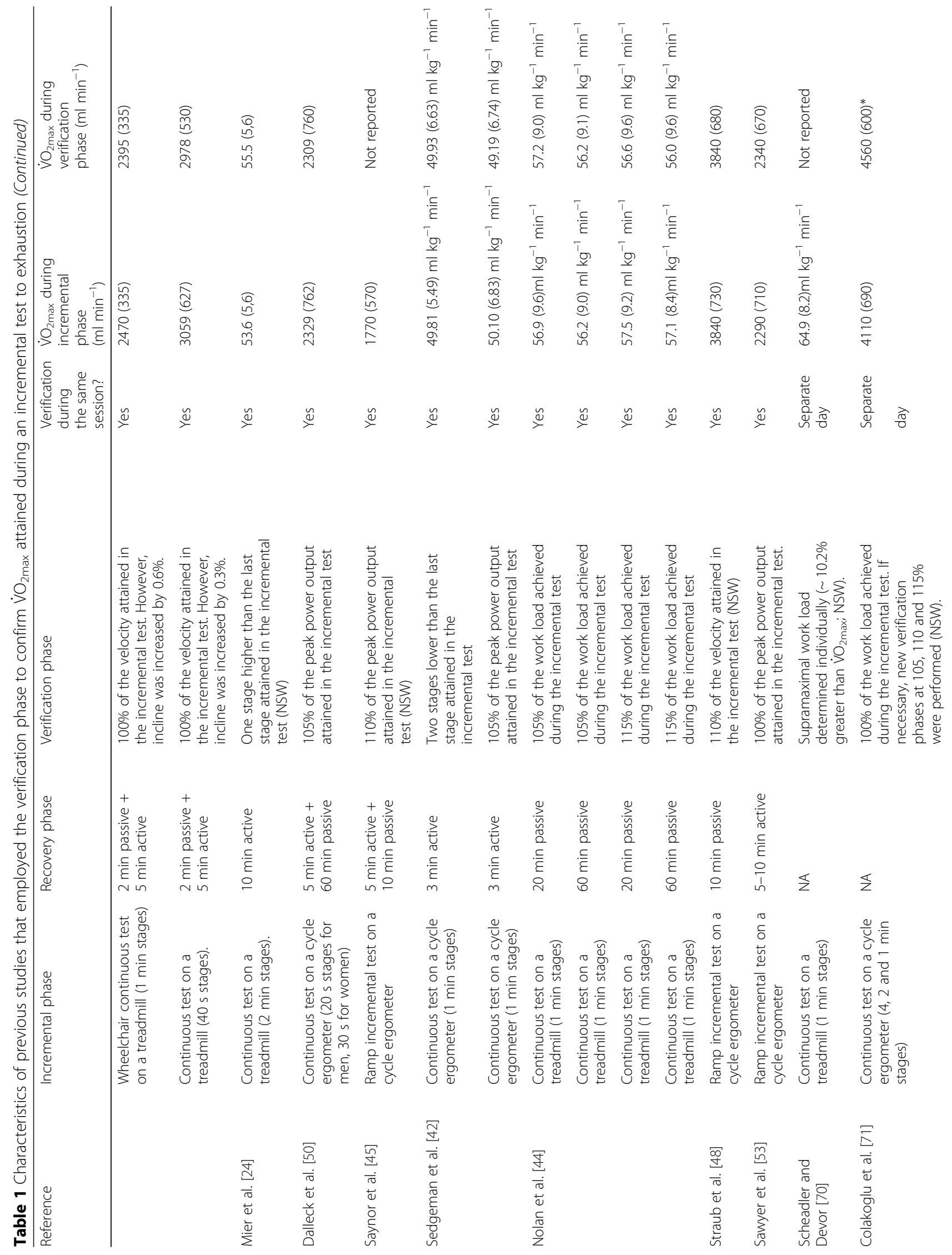




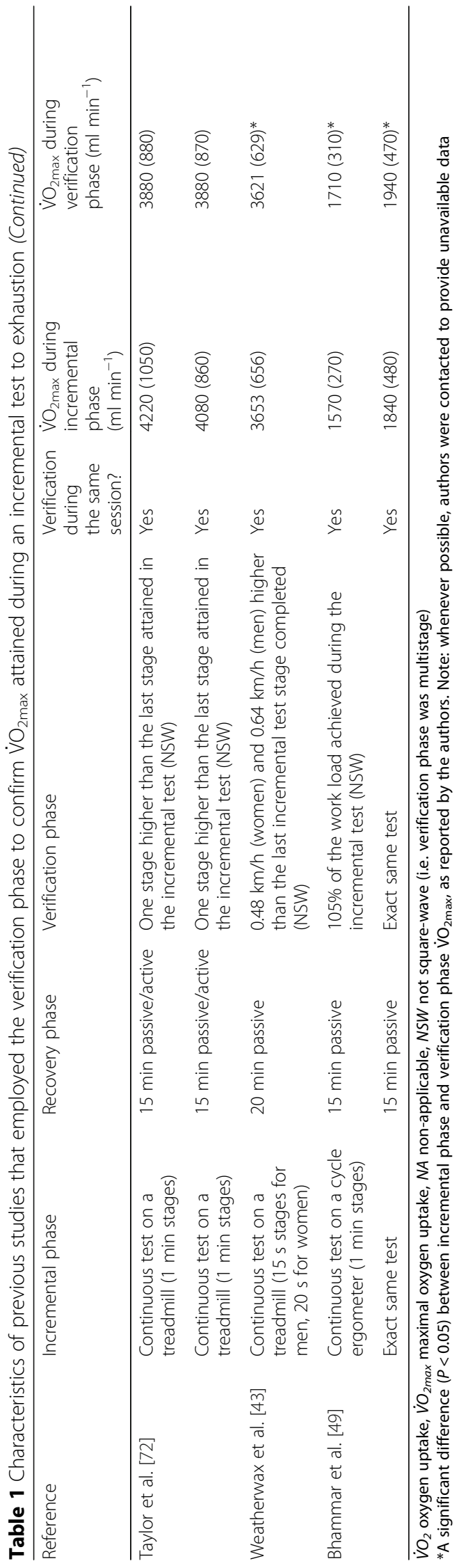


a
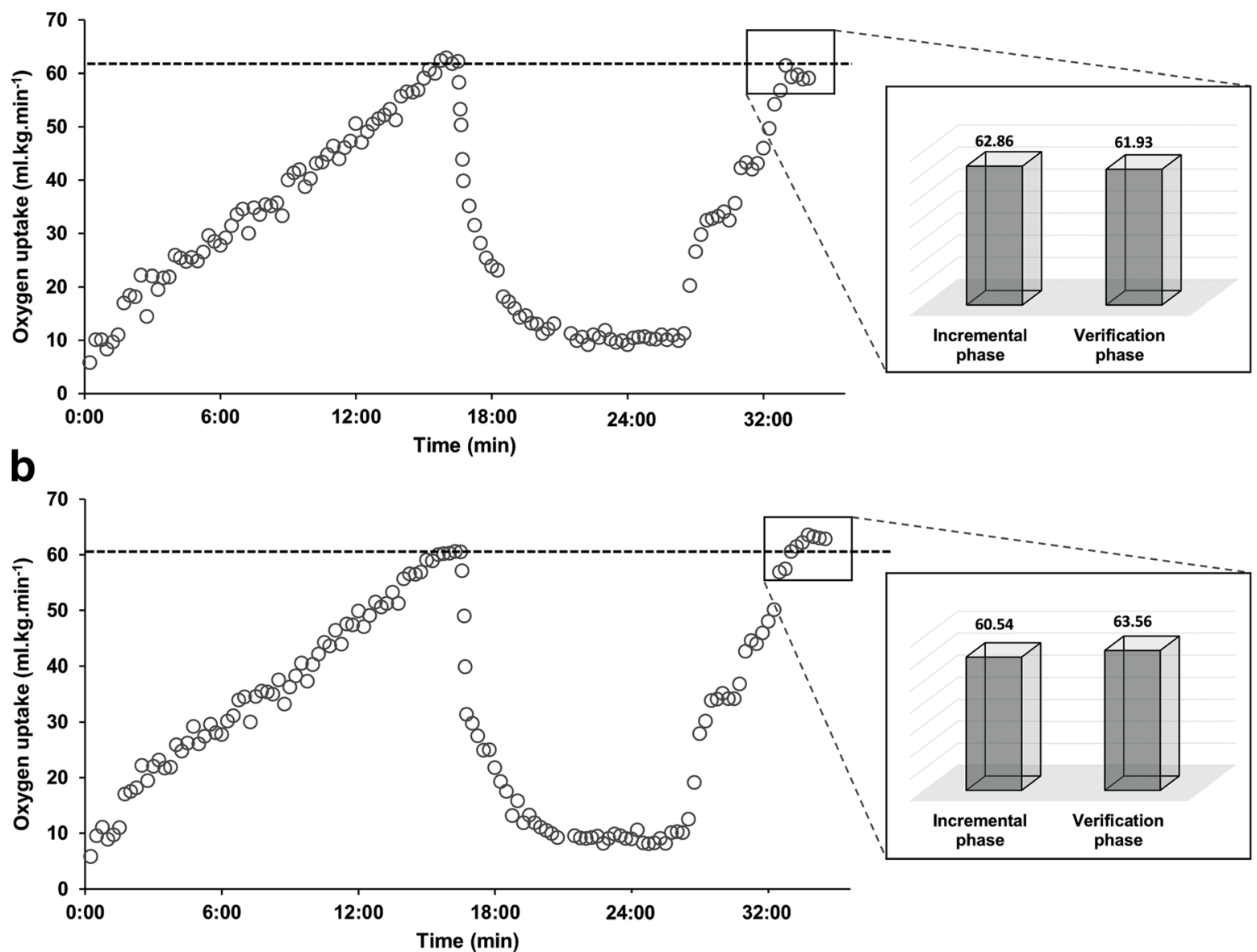

Fig. 2 Oxygen uptake $\left(\mathrm{V}_{2}\right)$ responses to an incremental test followed by a multistage verification phase for two representative subjects. a represents a valid test, while $\mathbf{b}$ represents a test were verification phase maximal oxygen uptake $\left(\dot{\mathrm{V}}_{2 \text { max }}\right)$ was higher than that reached during incremental phase, consequently, an invalid test. Note: differences between phases were considered as $\mathrm{V}_{2}$ differences $>2 \%$; $>3 \%$; $>2.2$ or $2.0 \mathrm{ml} \mathrm{kg}^{-1} \mathrm{~min}^{-1}$ and $\dot{\mathrm{V}}_{2}$ values presented as 15 -s averages

duration (i.e. $\leq 2 \mathrm{~min}$ ) may be somewhat "tight" for certain subjects, especially if associated with very high intensities, which may result in premature fatigue, as stated in the "Intensity" section.

A possible alternative is to perform multistage rather than square-wave verification phases (Fig. 1). Thus, subjects exercise at lower intensities before the workload corresponding to the verification phase is applied [36, 40]. This prior "warm-up stage" can enhance oxygen uptake, increasing the possibility to reach $\dot{\mathrm{VO}}_{2 \max }$ before exhaustion [37]. It was already shown that previous moderate and high-intensity exercises can increase $\mathrm{VO}_{2}$ kinetics $[55,56]$ and improve performance in supramaximal exercises [57]. As such, Midgley et al. [36] proposed a multistage verification phase comprising $2 \mathrm{~min}$ at $50 \%$ $\mathrm{iVO}_{2 \max }$ and $1 \mathrm{~min}$ at $70 \%$ followed by one stage higher than the last completed stage in the incremental test. Similarly, Scharhag-Rosenberger et al. [40] employed $1 \mathrm{~min}$ at $60 \% \mathrm{iVO}_{2 \max }$ followed by $110 \% \mathrm{iVO}_{2 \max }$ until exhaustion. Both protocols were able to confirm 90-95\% of the $\dot{\mathrm{V}}_{2 \max }$ identified at the incremental test. Accordingly, based on studies conducted so far (Table 1), it seems plausible to suggest that durations between 2 and 3 min should be adequate to confirm $\dot{\mathrm{VO}}_{2 \max }$ during verification phases, although some clinical populations may require longer periods. In addition, exercise mode should also be observed because subjects exercising on a cycle ergometer would be more susceptible to local muscle fatigue compared to treadmill running which, in turn, could lead to a shorter verification phase duration on the first of these (i.e. cycle ergometer).

\section{Recovery-How Long to Wait?}

Another factor that could possibly influence the verification phase is the time elapsed between incremental test and the verification phase. If a true maximal effort is provided by an individual during the incremental test, there will be a number of key perturbations to the skeletal 
muscle milieu, including depleted phosphocreatine stores coupled with increased metabolic acidosis. Performing the verification phase while inadequately recovered could lead to premature fatigue and inability to achieve a "true" $\dot{\mathrm{V}}_{2 \max }$. To the best of the author's knowledge, no study to date has investigated the influence of physiological outcome recovery on the $\dot{\mathrm{V}}_{2 \max }$ attained during the verification phase and, accordingly, this should be kept in mind when considering the following results.

Recovery periods of $10 \mathrm{~min}$ were the most frequently employed, although 5, 15, 20 and $60 \mathrm{~min}$ were also used (Table 1). Nolan et al. [44] found no difference (i.e. $\dot{\mathrm{V}} \mathrm{O}_{2 \max }> \pm 3 \%$ ) comparing 20 and 60 min recovery periods, recommending that $20 \mathrm{~min}$ should suffice for physically active subjects. Recently, it has been suggested that for healthy individuals, shorter recovery intervals (i.e. 5 or $10 \mathrm{~min}$ ) would already be sufficient [12]. Actually, 1and 3-min recovery periods have already been successfully employed in this population [41, 42, 51]. Therefore, as stated by Poole and Jones [12], recovery time between phases does not appear to be critical, although shorter periods may be more time-efficient. Notwithstanding, it is still necessary to experimentally confirm this in older adults and clinical populations. In addition, research investigating the relationship between the recovery of certain physiological responses (e.g. phosphocreatine and metabolic acidosis), the time between incremental test and verification phase and the $\dot{\mathrm{V}}_{2 \text { max }}$ achieved during the verification phase is also warranted.

\section{Data Processing}

Even if intensity, duration and recovery are adequately planned, inadequate data processing can interfere with the verification phase results and, ultimately, lead to flawed conclusions. Despite this, no study to date directly addressed the relationship between different sampling intervals and $\dot{\mathrm{V}} \mathrm{O}_{2 \max }$ attainment during the verification phase. Previous investigations have shown that both $\dot{\mathrm{V}}_{2 \max }$ and plateau incidence during an incremental test can differ according to the sampling interval employed. Specifically, Astorino et al. [22] found that 11-breath moving averages and 15-s averages of $\dot{\mathrm{VO}}_{2}$ data showed higher incidence of plateau (i.e. 100\%) compared to 30-s (57\%) and 60-s (8\%) averages. In addition, Astorino [10] demonstrated that 15- and 30-s averages, as well as breath-by-breath data, resulted in higher $\dot{\mathrm{V}} \mathrm{O}_{2 \max }$ values when compared to 60 -s averages, which was also observed by Robergs [58]. Consequently, Robergs et al. [30] suggested that "time-averaged sampling should be no longer than $30 \mathrm{~s}$ " while "breath-averaged sampling should be" performed by "15-breath running averages".

Thus, considering that the verification phase resembles an incremental discontinuous test, it could be suggested that $\dot{\mathrm{VO}}_{2}$ data from verification phases are processed through 15- to 30-s time intervals, for example, although some authors advocate that $15 \mathrm{~s}$ averages are preferable $[22,31]$. Since $15-\mathrm{s}$ averages may identify the highest $\mathrm{V}_{2 \max }$ [31] while also enhancing the incidence of plateaus in the incremental tests [22], it would allow researchers to use two criteria rather than just one to confirm $\dot{\mathrm{V}}_{2 \max }$. However, it is required that future studies address these questions and confirm whether these sampling intervals are best suited for the comparison of incremental and verification phases. Additionally, other data processing techniques such as digital filtering [30] should also be investigated.

\section{Validating the Verification Phase}

Professor Robert Pettitt once mentioned: "That is where art separates from science: at some point, you've got to pick a criterion". As previously mentioned, a true $\dot{\mathrm{VO}}_{2 \max }$ can be confirmed when incremental test and verification phase $\dot{\mathrm{VO}}_{2 \max }$ values do not differ. Nevertheless, what is or should be the appropriate cutoff point to define whether these results are similar? Some previous investigations compared the mean $\dot{\mathrm{V}}_{2 \max }$ between the two phases and when no statistical difference was identified, $\dot{\mathrm{V}} \mathrm{O}_{2 \max }$ was deemed true. However, concerns have been expressed about this procedure [11, 39, 59, 60]. Specifically, $\dot{\mathrm{V}}_{2 \max }$ comparisons through the mean values can mask a considerable between-trials variability in a few participants, even when no difference between the mean values is identified (i.e. $P>0.05$ ). Thus, the most appropriate procedure seems to be an individual approach $[11,36$, 39]. Midgley et al. [35] suggested that a difference $\leq 2 \%$, based on the equipment measurement error, would be an acceptable estimate. Notwithstanding, this criterion does not take into account $\dot{\mathrm{V}}_{2 \max }$ biological variability $[2,11]$, which may represent up to $90 \%$ of $\dot{V} \mathrm{O}_{2 \text { max }}$ total variability when assessed over different days [34]. Therefore, Kirkeberg et al. [41] suggested that differences $<3 \%$ were used to confirm $\dot{V} \mathrm{O}_{2 \max }$ in recreationally trained subjects, which could vary according to sampling rate and subjects' fitness level (see also Pettitt et al. [39]). In fact, based on the intra-subject coefficient of variation, several studies employed a 3\% cutoff point with some degree of success, being able to use it to confirm incremental test $\dot{\mathrm{VO}}_{2 \max }$ in healthy, active and trained subjects [42-44, 50, 61]. On the other hand, Saynor et al. [45] reported a 9\% intrasubject coefficient of variation in children with cystic fibrosis, showing that for some patient populations, less restrictive percentages may be necessary when comparing $\dot{\mathrm{V}} \mathrm{O}_{2 \max }$ between incremental and verification phases. Whichever the case, if an acute measurement is to be performed, there is a possibility that biological or day-to-day variability will not represent a substantial part of $\dot{\mathrm{VO}}_{2 \max }$ 
total variability as the time elapsed between incremental test and verification phase is usually short (i.e. few minutes). Consequently, short-term variability and equipment measurement error would be the main factors affecting this variability. However, if a chronic assessment is to be considered (e.g. pre-post a training intervention), short-term variability and equipment measurement error, but also day-to-day variability, may potentially influence the results. As only a few studies to date have employed the verification phase to confirm $\dot{\mathrm{V}} \mathrm{O}_{2 \max }$ in longitudinal studies, the validity of different criteria on this type of research remains to be tested.

Alternatively, Midgley et al. [36] proposed the utilization of the difference between the verification phase $\dot{\mathrm{VO}}_{2 \max }$ and the $\dot{\mathrm{V}} \mathrm{O}_{2 \max }$ modelled from a least-square linear regression based on the linear portion of the incremental test $\dot{\mathrm{VO}}_{2}$-workrate curve. Differences greater than $50 \%$ between measured and modelled values would indicate a plateau in the $\dot{\mathrm{VO}}_{2}$ and confirmation of a "true" $\dot{\mathrm{V}} \mathrm{O}_{2 \max }$. According to the authors, the advantage of this criterion would be that it is specific to subject and test characteristics (e.g. protocol, ergometer). Notwithstanding, the 50\% value was based on an arbitrary choice and, therefore, needs further investigation although the rationale seems reasonable [36]. Thus, there seems to be no consensus as to the best individual-based criterion to be used to validate verification phase $\dot{\mathrm{VO}}_{2 \max }$. Accordingly, although the verification phase "science" is differentiating itself from "art", criteria need to be based on the highest category of scientific evidence and, therefore, a great effort is still needed before a consensus is found. Future studies comparing different criteria may help shed some light on this topic. In addition, studies should, whenever possible, make clear what criteria were used and why they were chosen, as well as report reliability measures such as $\dot{\mathrm{VO}}_{2 \max }$ typical errors and coefficients of variation [62]. It is also advisable that scientists should develop these criteria in their laboratories rather than using values developed by other laboratories with different equipment and methodologies.

\section{Additional Considerations}

To rely on a single incremental test and verification phase protocol to meet all characteristics of a wide range of populations is somewhat over simplistic or naïve. Therefore, based on a pragmatic perspective, a plausible proposal would be to structure the incremental protocol according to the variables that will be assessed (e.g. $\dot{\mathrm{V}} \mathrm{O}_{2 \max }$, lactate threshold, percentage of HR associated to the ventilatory threshold). Verification phase, in turn, would be designed based on the incremental test protocol, as discussed in sections "Verification Phase: a Light at the End of the Tunnel?" and "How It Should Be Done-Is There Consensus?". Accordingly, it is important that authors clearly present their reasons for choosing that specific verification phase. Moreover, one major advantage of the verification phase is that it deals with the same unit as the incremental test (i.e. $1 \mathrm{~min}^{-1}$ or $\mathrm{ml} \mathrm{kg}^{-1} \mathrm{~min}^{-1}$ ). Thus, when a higher $\dot{\mathrm{VO}}_{2 \max }$ is identified in the verification phase and it is not possible to perform a new incremental test and/or verification phase, for example, researchers are provided with the highest $\dot{\mathrm{VO}}_{2}$ value reached, which would not be possible through other criteria based solely on a single incremental test (Fig. 2b).

In fact, this raises an important methodological question: "What actually is $\dot{\mathrm{V}}{ }_{2 \max }$ after performing the incremental test and the verification phase?". The majority of studies to date found both incremental test and verification phase $\dot{\mathrm{V}}_{2 \max }$ to be similar (Table 1), but did not present a clear suggestion as to which value was to be selected. Possibilities are that the $\mathrm{VO}_{2 \max }$ value from the incremental test, the verification phase (possibly the higher) or the averaged value from both may be selected. Because both phases need to present sufficiently similar $\dot{\mathrm{V}} \mathrm{O}_{2 \max }$ results to validate the test (as explained in section "Validating the Verification Phase"), it may be plausible to suggest that there should be no substantial interference on $\dot{\mathrm{VO}}_{2 \max }$ results irrespective of which $\dot{\mathrm{V}} \mathrm{O}_{2 \max }$ value is selected, although this should be clearly stated in the manuscript. Finally, it may also be possible for a subject to terminate the two phases at submaximal efforts resulting in similar $\dot{\mathrm{V}} \mathrm{O}_{2 \max }$ values in both phases. Even though it may happen, it is unlikely to occur, especially if the verification phase is performed with an adequate duration (see the "Duration" section). Actually, this is exactly what favors the verification phase as it requires this submaximal value to happen twice and not only once as in an ordinary incremental test.

\section{Is It Safe?}

Because subjects need to perform two efforts to the point of exhaustion, researchers may worry about possible complications arriving from the extra effort. When considering all studies presented in Table 1, a total of approximately 834 subjects comprising healthy children, adults and elderly, athletes, overweight/obese and patient populations were assessed. Hawkins et al. [32] performed a total of 156 verification phases at 130\% $\mathrm{iVO}_{2 \max }$ in well-trained runners. According to the authors, no adverse events occurred during verification phases. Nevertheless, it may be suggested that as the subjects performed the verification phase the next day, this result should not be extrapolated to phases performed in the same session. In this regard, physically active subjects and athletes submitted to a verification phase 1 and $3 \mathrm{~min}$ after the incremental test, respectively, also did not present any complication [51]. This 
result is also corroborated by other studies [41, 42], including sedentary and untrained subjects $[31,40]$ and also children [49].

Recently, the possibility that, at least in a clinical setting, the verification phase would be "unrealistic and unethical in certain patient populations" was raised [63]. When only those studies that assessed special populations are taken into account (Table 1), out of $\sim 241$ subjects evaluated, only three cases related to the verification phase were reported. One obese subject and another with chronic heart failure requested the verification phase not to be performed [53, 64], whereas only one chronic heart failure patient did not perform his verification phase due to the "onset of runs of multifocal ectopic beats" [53]. Thus, provided that all safety measures are taken, the verification phase seems to be well tolerated in both healthy and patient populations in general $[12,65]$.

\section{Future Directions}

It is clear that the verification phase can be a practical and sensitive method to confirm a maximal incremental test $\dot{\mathrm{V}} \mathrm{O}_{2 \max }$. However, there are still some issues that deserve to be addressed. Specifically, to the best of the author's knowledge, no study has sought to identify a verification protocol that is feasible for different populations (e.g. healthy adults and elderly). Such a protocol could allow a better comparison of verification phase $\dot{\mathrm{V}}_{2 \max }$ across different studies, and an adequate comparison of other outcomes assessed as well (e.g. maximal power output, ventilatory thresholds). It could also assist researchers to investigate the effects of an outcome on different populations' $\dot{\mathrm{VO}}_{2 \max }$. As an example, the effect of aging on $\dot{\mathrm{V}} \mathrm{O}_{2 \max }$ can be studied based on a protocol that is feasible for both adults and elderly subjects and that does not rely on secondary criteria or peak $\mathrm{VO}_{2}$.

Furthermore, comparisons between square-wave and multistage verification protocols can also enhance knowledge on the applicability of these models, especially in patient populations who could benefit from the possibility to enhance their $\dot{V} \mathrm{O}_{2}$ kinetics and time to exhaustion. Additionally, current criteria used to compare the verification phase and incremental test $\dot{\mathrm{V}} \mathrm{O}_{2 \max }$ still warrant further investigation. Likewise, as $\dot{\mathrm{VO}}_{2 \max }$ should be confirmed on an individual basis, mean value comparisons between submaximal and supramaximal verification phases may not be the ideal approach and future studies may seek to examine and compare the differences between these protocols based on individual differences.

Recently, Astorino et al. [66] compared $\dot{\mathrm{VO}}_{2 \max }$ after 20 sessions of high-intensity interval training in three different groups. The authors reported that $\dot{\mathrm{VO}}_{2 \max }$ was enhanced between 8.9 and $12.3 \%$ in the three groups and that results from the verification phases suggested "that participants did exhibit 'true' $\dot{\mathrm{V}} \mathrm{O}_{2 \max }$ and that" their "reported increases in $\dot{\mathrm{V}}_{2 \max }$ are repeatable and not due to random error". Therefore, experimental studies employing verification phases are also warranted in order to identify if it can provide any further information or how does it impact the outcomes compared to the incremental test alone. Last but not least, investigations aimed at assessing the verification phase as well as those experiments employing it should consider using the keyword "verification phase" to help researchers retrieve these studies more easily.

\section{Conclusions}

Although the plateau is still considered the best evidence to confirm $\dot{\mathrm{VO}}_{2 \max }$, its frequency is or may be low. Moreover, secondary criteria, at least those mentioned in the present review, lack sensitivity and reliability to confirm $\dot{\mathrm{V}} \mathrm{O}_{2 \max }$, which lead authors to suggest its complete rejection. On the other hand, as discussed throughout this review, the verification phase was demonstrated as a practical and sensitive method to confirm $\dot{\mathrm{VO}}_{2 \max }$ among different populations. Accordingly, both supramaximal and submaximal protocols appear to be suitable as long as the incremental test design is taken in consideration, while verification phase durations between 2 and 3 min should already be adequate for most, but not all, subjects. As suggested by previous researchers, recovery between incremental and verification phases does not appear to be critical, although this recommendation still requires further investigation in older adults and clinical populations. Further, despite not being addressed directly during verification phases, processing data with 15- to 30-s averages seems reasonable, while 15$\mathrm{s}$ averages may also enhance plateau incidences. Additionally, incremental and verification phase $\dot{\mathrm{VO}}_{2}$ comparisons should be performed on an individual basis and not based on group means comparisons. In this regard, whenever possible researchers should provide reliability measures such as coefficients of variation and typical errors. Finally, as already mentioned, researchers, physiologists and clinicians must keep in mind that the main purpose of the verification phase is not simply to achieve a $\dot{\mathrm{VO}}_{2}$ similar to the incremental phase, but to create a platform that enables a higher $\dot{\mathrm{VO}}_{2}$ to be reached if it has not been reached previously.

\section{Additional File}

Additional file 1: Table S1. Incidence of $\dot{\mathrm{V}} \mathrm{O}_{2 \max }$ deemed valid

based on the verification phase as well as phase duration and method employed for comparison between phases along other criteria employed. (PDF 316 kb)

\section{Acknowledgements}

The author would like to thank Dr. Eurico Wilhelm for his valuable and pertinent comments on the manuscript. 


\section{Funding}

No sources of funding were used to assist in the preparation of this article.

\section{Availability of Data and Materials}

The datasets supporting the conclusions of this article are included within the article and its supplementary file.

\section{Ethics Approval and Consent to Participate}

Not applicable.

\section{Consent for Publication}

Not applicable.

\section{Competing Interests}

Gustavo Schaun declares that he has no conflicts of interest relevant to the content of this review.

\section{Publisher's Note}

Springer Nature remains neutral with regard to jurisdictional claims in published maps and institutional affiliations.

Received: 15 August 2017 Accepted: 19 November 2017

Published online: 08 December 2017

\section{References}

1. Hill AV, Lupton H. Muscular exercise, lactic acid, and the supply and utilization of oxygen. QJM. 1923;0s-16(62):135-71.

2. Beltz NM, Gibson AL, Janot JM, Kravitz L, Mermier CM. Graded exercise testing protocols for the determination of VO2max: historical perspectives, progress, and future considerations. J Sports Med. 2016;2016:3968393.

3. Kodama S, Saito K, Tanaka S, Maki M, Yachi Y, Asumi M, et al. Cardiorespiratory fitness as a quantitative predictor of all-cause mortality and cardiovascular events in healthy men and women: a meta-analysis. JAMA. 2009:301(19):2024-35.

4. Lavie CJ, Arena R, Swift DL, Johannsen NM, Sui X, Lee DC, et al. Exercise and the cardiovascular system: clinical science and cardiovascular outcomes. Circ Res. 2015;117(2):207-19.

5. McAuley PA, Blaha MJ, Keteyian SJ, Brawner CA, Al Rifai M, Dardari ZA, et al. Fitness, fatness, and mortality: the FIT (Henry Ford exercise testing) project. Am J Med. 2016;129(9):960-5.e1.

6. Joyner MJ, Coyle EF. Endurance exercise performance: the physiology of champions. J Physiol. 2008;586(1):35-44

7. Aziz AR, Chia M, Teh KC. The relationship between maximal oxygen uptake and repeated sprint performance indices in field hockey and soccer players. J Sports Med Phys Fitness. 2000;40(3):195-200.

8. Girard O, Mendez-Villanueva A, Bishop D. Repeated-sprint ability-part I: factors contributing to fatigue. Sports Med. 2011:41(8):673-94.

9. Albouaini K, Egred M, Alahmar A, Wright DJ. Cardiopulmonary exercise testing and its application. Heart. 2007:93(10):1285-92.

10. Astorino TA. Alterations in VOmax and the VO plateau with manipulation of sampling interval. Clin Physiol Funct Imaging. 2009;29(1):60-7.

11. Midgley AW, Carroll S. Emergence of the verification phase procedure for confirming 'true' VO(2max). Scand J Med Sci Sports. 2009;19(3):313-22.

12. Poole DC, Jones AM. Measurement of the maximum oxygen uptake VO2max: VO2peak is no longer acceptable. J Appl Physiol (1985). 2017; 122(4):997-1002.

13. Howley ET, Bassett DR Jr, Welch HG. Criteria for maximal oxygen uptake: review and commentary. Med Sci Sports Exerc. 1995:27(9):1292-301.

14. Midgley AW, McNaughton LR, Polman R, Marchant D. Criteria for determination of maximal oxygen uptake: a brief critique and recommendations for future research. Sports Med. 2007;37(12):1019-28.

15. Misquita NA, Davis DC, Dobrovolny CL, Ryan AS, Dennis KE, Nicklas BJ. Applicability of maximal oxygen consumption criteria in obese, postmenopausal women. J Womens Health Gend Based Med. 2001;10(9):879-85.

16. Niemelä K, Palatsi I, Linnaluoto $M$, Takkunen J. Criteria for maximum oxygen uptake in progressive bicycle tests. Eur J Appl Physiol Occup Physiol. 1980; 44(1):51-9.

17. Poole DC, Wilkerson DP, Jones AM. Validity of criteria for establishing maximal O2 uptake during ramp exercise tests. Eur J Appl Physiol. 2008; 102(4):403-10.
18. Thoden JS. Testing aerobic power. In: MacDougall JD, Wenger HA, Green $\mathrm{HJ}$, editors. Physiological testing of the high-performance athlete. Champaign: Human Kinetics; 1991. p. 107-73.

19. Hill AV, Long CNH, Lupton $\mathrm{H}$. Muscular exercise, lactic acid and the supply and utilization of oxygen. Proc Royal Soc B. 1924;97(682):155-76.

20. Noakes TD. How did a V Hill understand the VO2max and the "plateau phenomenon"? Still no clarity? Br J Sports Med. 2008;42(7):574-80.

21. Taylor HL, Buskirk E, Henschel A. Maximal oxygen intake as an objective measure of cardio-respiratory performance. J Appl Physiol. 1955;8(1):73-80.

22. Astorino TA, Robergs RA, Ghiasvand F, Marks D, Burns S. Incidence of the oxygen plateau at VO2max during exercise testing to volitional fatigue. J Exerc Physiol Online. 2000;3(4):12

23. Froelicher VF Jr, Brammell H, Davis G, Noguera I, Stewart A, Lancaster MC. A comparison of three maximal treadmill exercise protocols. J Appl Physiol. 1974;36(6):720-5.

24. Mier CM, Alexander RP, Mageean AL. Achievement of VO2max criteria during a continuous graded exercise test and a verification stage performed by college athletes. J Strength Cond Res. 2012;26(10):2648-54.

25. Cumming GR, Friesen W. Bicycle ergometer measurement of maximal oxygen uptake in children. Can J Physiol Pharmacol. 1967;45(6):937-46.

26. Howley ET. VO2max and the plateau-needed or not? Med Sci Sports Exerc. 2007;39(1):101-2. https://doi.org/10.1249/mss.0b013e31802dc897.

27. Lucia A, Rabadan M, Hoyos J, Hernandez-Capilla M, Perez M, San Juan AF, et al. Frequency of the $\mathrm{VO} 2$ max plateau phenomenon in world-class cyclists. Int J Sports Med. 2006;27(12):984-92.

28. Day JR, Rossiter HB, Coats EM, Skasick A, Whipp BJ. The maximally attainable VO2 during exercise in humans: the peak vs. maximum issue. J Appl Physio (1985). 2003:95(5):1901-7.

29. Rossiter HB, Kowalchuk JM, Whipp BJ. A test to establish maximum $\mathrm{O} 2$ uptake despite no plateau in the $\mathrm{O} 2$ uptake response to ramp incremental exercise. J Appl Physiol (1985). 2006;100(3):764-70.

30. Robergs RA, Dwyer D, Astorino T. Recommendations for improved data processing from expired gas analysis indirect calorimetry. Sports Med. 2010:40(2):95-111.

31. Astorino TA, White AC, Dalleck LC. Supramaximal testing to confirm attainment of VO2max in sedentary men and women. Int J Sports Med. 2009;30(4):279-84

32. Hawkins MN, Raven PB, Snell PG, Stray-Gundersen J, Levine BD. Maximal oxygen uptake as a parametric measure of cardiorespiratory capacity. Med Sci Sports Exerc. 2007:39(1):103-7.

33. Brink-Elfegoun T, Holmberg HC, Ekblom MN, Ekblom B. Neuromuscular and circulatory adaptation during combined arm and leg exercise with different maximal work loads. Eur J Appl Physiol. 2007;101(5):603-11.

34. Katch VL, Sady SS, Freedson P. Biological variability in maximum aerobic power. Med Sci Sports Exerc. 1982;14(1):21-5.

35. Midgley AW, McNaughton LR, Carroll S. Verification phase as a useful tool in the determination of the maximal oxygen uptake of distance runners. Appl Physiol Nutr Metab. 2006;31(5):541-8.

36. Midgley AW, Carroll S, Marchant D, McNaughton LR, Siegler J. Evaluation of true maximal oxygen uptake based on a novel set of standardized criteria. Appl Physiol Nutr Metab. 2009;34(2):115-23.

37. Midgley AW, McNaughton LR, Carroll S. Time at VO2max during intermittent treadmill running: test protocol dependent or methodological artefact? Int J Sports Med. 2007;28(11):934-9.

38. Morgan DW, Baldini FD, Martin PE, Kohrt WM. Ten kilometer performance and predicted velocity at VO2max among well-trained male runners. Med Sci Sports Exerc. 1989;21(1):78-83.

39. Pettitt RW, Clark IE, Ebner SM, Sedgeman DT, Murray SR. Gas exchange threshold and VO2max testing for athletes: an update. J Strength Cond Res. 2013;27(2):549-55

40. Scharhag-Rosenberger F, Carlsohn A, Cassel M, Mayer F, Scharhag J. How to test maximal oxygen uptake: a study on timing and testing procedure of a supramaximal verification test. Appl Physiol Nutr Metab. 2011:36(1):153-60

41. Kirkeberg JM, Dalleck LC, Kamphoff CS, Pettitt RW. Validity of 3 protocols for verifying VO2 max. Int J Sports Med. 2011;32(4):266-70.

42. Sedgeman D, Dalleck L, Clark IE, Jamnick N, Pettitt RW. Analysis of squarewave bouts to verify VO2max. Int J Sports Med. 2013;34(12):1058-62.

43. Weatherwax RM, Richardson TB, Beltz NM, Nolan PB, Dalleck L. Verification testing to confirm VO2max in altitude-residing, endurance-trained runners. Int J Sports Med. 2016;37(7):525-30. 
44. Nolan PB, Beaven ML, Dalleck L. Comparison of intensities and rest periods for VO2max verification testing procedures. Int J Sports Med. 2014;35(12):1024-9.

45. Saynor ZL, Barker AR, Oades PJ, Williams CA. A protocol to determine valid VO2max in young cystic fibrosis patients. J Sci Med Sport. 2013; 16(6):539-44.

46. Saynor ZL, Barker AR, Oades PJ, Williams CA. Reproducibility of maximal cardiopulmonary exercise testing for young cystic fibrosis patients. J Cyst Fibros. 2013;12(6):644-50.

47. Morton RH. Why peak power is higher at the end of steeper ramps: an explanation based on the "critical power" concept. J Sports Sci. 2011; 29(3):307-9.

48. Straub AM, Midgley AW, Zavorsky GS, Hillman AR. Ramp-incremented and RPE-clamped test protocols elicit similar VO2max values in trained cyclists. Eur J Appl Physiol. 2014;114(8):1581-90.

49. Bhammar DM, Stickford JL, Bernhardt V, Babb TG. Verification of maximal oxygen uptake in obese and nonobese children. Med Sci Sports Exerc. 2017; 49(4):702-10.

50. Dalleck LC, Astorino TA, Erickson RM, McCarthy CM, Beadell AA, Botten BH. Suitability of verification testing to confirm attainment of $\mathrm{VO}(2) \mathrm{max}$ in middle-aged and older adults. Res Sports Med. 2012;20(2):118-28.

51. Foster C, Kuffel E, Bradley N, Battista RA, Wright G, Porcari JP, et al. VO2max during successive maximal efforts. Eur J Applied Physiol. 2007;102(1):67-72.

52. Leicht CA, Tolfrey K, Lenton JP, Bishop NC, Goosey-Tolfrey VL. The verification phase and reliability of physiological parameters in peak testing of elite wheelchair athletes. Eur J Appl Physiol. 2013;113(2):337-45.

53. Sawyer BJ, Tucker WJ, Bhammar DM, Gaesser GA. Using a verification test for determination of VO2max in sedentary adults with obesity. J Strength Cond Res. 2015;29(12):3432-8.

54. Poole DC, Jones AM. Oxygen uptake kinetics. Compr Physiol. 2012;2(2):933-96.

55. Faisal A, Beavers KR, Robertson AD, Hughson RL. Prior moderate and heavy exercise accelerate oxygen uptake and cardiac output kinetics in endurance athletes. J Appl Physiol (1985). 2009;106(5):1553-63.

56. Gurd BJ, Peters SJ, Heigenhauser GJ, LeBlanc PJ, Doherty TJ, Paterson DH, et al. Prior heavy exercise elevates pyruvate dehydrogenase activity and speeds $\mathrm{O} 2$ uptake kinetics during subsequent moderate-intensity exercise in healthy young adults. J Physiol. 2006;577(Pt 3):985-96.

57. Jones AM, Wilkerson DP, Burnley M, Koppo K. Prior heavy exercise enhances performance during subsequent perimaximal exercise. Med Sci Sports Exerc. 2003;35(12):2085-92

58. Robergs RA. An exercise physiologist's "contemporary" interpretations of the "ugly and creaking edifices" of the VO2max concept. J Exerc Physiol Online. 2001;4(1):44

59. Hecksteden A, Kraushaar J, Scharhag-Rosenberger F, Theisen D, Senn S, Meyer T. Individual response to exercise training - a statistical perspective. J Appl Physiol (1985). 2015;118(12):1450-9.

60. Noakes TD. Maximal oxygen uptake as a parametric measure of cardiorespiratory capacity: comment. Med Sci Sports Exerc. 2008;40(3):585. author reply 6

61. Astorino TA, White AC. Assessment of anaerobic power to verify VO2max attainment. Clin Physiol Funct Imaging. 2010;30(4):294-300.

62. Hopkins WG. Measures of reliability in sports medicine and science. Sports Med. 2000;30(1):1-15.

63. van Breda E, Schoffelen PFM. Clinical VO2peak is "part of the deal". J Appl Physiol (1985). 2017;122(5):1370

64. Bowen TS, Cannon DT, Begg G, Baliga V, Witte KK, Rossiter HB. A novel cardiopulmonary exercise test protocol and criterion to determine maximal oxygen uptake in chronic heart failure. J Appl Physiol (1985). 2012;113(3):451-8.

65. Poole DC, Jones AM. Reply to Drs. Van Breda et al. J Appl Physiol (1985). 2017;122(5):1371-2.

66. Astorino TA, Edmunds RM, Clark A, King L, Gallant RA, Namm S, et al. Highintensity interval training increases cardiac output and VO2max. Med Sci Sports Exerc. 2017:49(2):265-73.

67. Stachenfeld NS, Eskenazi M, Gleim GW, Coplan NL, Nicholas JA. Predictive accuracy of criteria used to assess maximal oxygen consumption. Am Heart J. 1992;123(4):922-5

68. Wood RE, Hills AP, Hunter GR, King NA, Byrne NM. VO2max in overweight and obese adults: do they meet the threshold criteria? Med Sci Sports Exerc. 2010;42(3):470-7.
69. Beltrami FG, Froyd C, Mauger AR, Metcalfe AJ, Marino F, Noakes TD. Conventional testing methods produce submaximal values of maximum oxygen consumption. Br J Sports Med. 2012;46:23-9.

70. Scheadler CM, Dever ST. VO2max measured with a self-selected work rate protocol on an automated treadmill. Med Sci Sports Exerc. 2015;47(10): 2158-65.

71. Colakoglu M, Ozkaya O, Balci GA, Yapiciogly B. Stroke volume responses may be related to the gap between peak and maximal $\mathrm{O} 2$ consumption. Isokinet Exerc Sci. 2016;24:133-9.

72. Taylor K, Seegmiller J, Vella CA. The decremental protocol as an alternative protocol to measure VO2max in athletes. Int J Sports Physiol Perform. 2016; 11(8):1094-9.

\section{Submit your manuscript to a SpringerOpen ${ }^{\circ}$ journal and benefit from:}

- Convenient online submission

- Rigorous peer review

- Open access: articles freely available online

- High visibility within the field

- Retaining the copyright to your article 